\title{
Differential diagnosis of Bartter syndrome, Gitelman syndrome, and pseudo-Bartter/Gitelman syndrome based on clinical characteristics
}

\author{
Natsuki Matsunoshita, MD1', Kandai Nozu, MD, PhD1', Akemi Shono, PhD1, \\ Yoshimi Nozu, MNS'1, Xue Jun Fu, MD, PhD1, Naoya Morisada, MD, PhD1, \\ Naohiro Kamiyoshi, MD1, Hiromi Ohtsubo, MD1, Takeshi Ninchoji, MD, PhD1, \\ Shogo Minamikawa, MD1, Tomohiko Yamamura, MD¹, Koichi Nakanishi, MD, PhD², \\ Norishige Yoshikawa, MD, PhD², Yuko Shima, MD, PhD², Hiroshi Kaito, MD, $\mathrm{PhD}^{1}$ and \\ Kazumoto lijima, MD, $\mathrm{PhD}^{1}$
}

\begin{abstract}
Purpose: Phenotypic overlap exists among type III Bartter syndrome (BS), Gitelman syndrome (GS), and pseudo-BS/GS (p-BS/ GS), which are clinically difficult to distinguish. We aimed to clarify the differences between these diseases, allowing accurate diagnosis based on their clinical features.
\end{abstract}

Methods: A total of 163 patients with genetically defined type III BS $(n=30)$, GS $(n=90)$, and p-BS/GS $(n=43)$ were included. Age at diagnosis, sex, body mass index, estimated glomerular filtration rate, and serum and urine electrolyte concentrations were determined.

Results: Patients with p-BS/GS were significantly older at diagnosis than those with type III BS and GS. Patients with p-BS/GS included a significantly higher percentage of women and had a lower body mass index and estimated glomerular filtration rate than did patients with GS. Although hypomagnesemia and hypocalciuria were predominant biochemical findings in patients with GS, 17 and 23\% of patients with type III BS and p-BS/GS, respectively, also showed these abnormalities. Of patients with type III BS, GS, and p-BS/GS, 40, 12, and $63 \%$, respectively, presented with chronic kidney disease.

Conclusions: This study clarified the clinical differences between BS, GS, and p-BS/GS for the first time, which will help clinicians establish differential diagnoses for these three conditions.

Genet Med advance online publication 16 April 2015

Key Words: Bartter syndrome; Gitelman syndrome; pseudoBartter; pseudo-Gitelman; salt-losing tubulopathy

\section{INTRODUCTION}

Bartter syndrome (BS) (OMIM \#s: type I, 601678; type II, 241200; type III, 607364; type IV, 602522; type IVb, 613090) and Gitelman syndrome (GS) (OMIM \# 263800) are inherited autosomal-recessive, salt-losing tubulopathies characterized by hypokalemic metabolic alkalosis. BS and GS are reportedly caused by mutations in genes encoding ion transporters or channels, leading directly or indirectly to loss of function. ${ }^{1-6}$ These genes include $S L C 12 A 1$, which encodes the apical furosemide-sensitive $\mathrm{Na}-\mathrm{K}-2 \mathrm{Cl}$ cotransporter ${ }^{2}$; KCNJ1, which encodes the apical renal outer medullary potassium channel; $C L C N K B$, which encodes the basolateral chloride channel $\mathrm{Kb}$ (expressed in the thick ascending limb of Henle's loop and in the distal convoluted tubule) $)^{1,7}$; and BSND, which encodes barttin, a subunit of chloride channels $\mathrm{Ka}$ and $\mathrm{Kb}$. Mutations in these genes lead to types I-IV BS, respectively. ${ }^{6,8}$ Combined mutations in both CLCNKA and CLCNKB result in type IVb BS. ${ }^{9,10}$ By contrast, mutations in the SLC12A3 gene, which encodes the apical thiazide-sensitive $\mathrm{Na}-\mathrm{Cl}$ cotransporter (NCCT) in the distal convoluted tubule, are responsible for GS. ${ }^{4}$ Types I, II, IV, and IVb BS (antenatal BS) usually present during the neonatal period with relatively severe symptoms, whereas type III BS (classic BS) presents during early childhood with milder symptoms. In contrast to BS, GS is usually diagnosed during late childhood or adulthood. However, phenotypic overlap frequently occurs between type III BS and GS, which are difficult to diagnose based on their clinical presentations and require genetic tests. For example, some patients with type III BS may show clinical features of GS, including hypomagnesemia and hypocalciuria, and diuretic tests may fail to differentiate between them. ${ }^{711-13}$ Moreover, mutations in known disease-related genes have not been identified in some patients with clinically diagnosed BS/GS. This suggests that some acquired conditions may cause a BS/GS-like disorder, or pseudo-BS/GS (p-BS/GS), associated with loss of sodium or chloride in the urine, stool, or vomitus or with chloride-intake deficiency, resulting in clinical symptoms identical to those of BS/GS. As previously reported, p-BS/GS may be caused by a wide variety of conditions such

${ }^{1}$ Department of Pediatrics, Kobe University Graduate School of Medicine, Kobe, Japan; ${ }^{2}$ Department of Pediatrics, Wakayama Medical University, Wakayama, Japan. Correspondence: Kandai Nozu (nozu@med.kobe-u.ac.jp)

Submitted 1 November 2014; accepted 17 March 2015; advance online publication 16 April 2015. doi:10.1038/gim.2015.56 
as surreptitious diuretic use, laxative abuse, a chronic chloridedeficient diet, cyclic vomiting, congenital chloride diarrhea, and cystic fibrosis. ${ }^{5}$ Despite the need for accurate diagnosis of these three diseases, the differences in their clinical characteristics have rarely been analyzed, and few useful indicators currently exist. This study aimed to clarify the clinical differences among patients with genetically defined type III BS, GS, and p-BS/GS.

\section{MATERIALS AND METHODS}

\section{Ethical considerations}

This study was approved by the institutional review board of Kobe University Graduate School of Medicine. Informed consent was obtained from all patients or their parents.

\section{Inclusion criteria}

Clinical and laboratory findings of patients with a clinical diagnosis of BS/GS were retrospectively obtained from their medical records. All patients in this study had low serum potassium concentrations and metabolic alkalosis. Hypomagnesemia was defined as a serum magnesium concentration $<1.7 \mathrm{mg} / \mathrm{dl}$. Hypocalciuria was defined as a urinary calcium-tocreatinine ratio $<0.04 \mathrm{mg} / \mathrm{mg}$. Body height, SD of body height, body weight, body mass index (BMI), serum creatinine, and estimated glomerular filtration rate (eGFR) were obtained at the time of mutational analysis. eGFR was used as an indicator of renal function and was calculated using the formula developed by Schwartz et al. ${ }^{14,15}$ or equations designed to obtain the eGFR in Japanese individuals ${ }^{16}$ for patients aged $\leq 17$ and $\geq 18$ years, respectively. Urinary electrolyte excretion was evaluated as the fractional excretion of sodium (FENa) and the fractional excretion of chloride (FECl).

\section{Mutational analyses}

Genomic DNA was isolated from peripheral blood leukocytes of the patients and their family members using a compact extraction system for DNA isolation (QuickGene-Mini80; Fujifilm, Tokyo, Japan), according to the manufacturer's instructions. All exons and exon-intron boundaries of the CLCNKB and SLC12A3 genes were amplified by polymerase chain reaction and direct sequencing using previously described primer pairs. $^{10,17}$ Patients suspected to have BS/GS with no CLCNKB or SLC12A3 mutations were diagnosed as having p-BS/GS. If patients suspected to have BS/GS carried only one CLCNKB or SLC12A3 mutant allele, we performed additional semiquantitative polymerase chain reaction ${ }^{10,18}$ or multiplex ligation-dependent probe amplification using the SALSA P266CLCNKB or P136-SLC12A3 multiplex ligation-dependent probe amplification assays (MRC-Holland, Amsterdam, The Netherlands) to detect large heterozygous deletions. Total RNA from leukocytes and/or urine sediment was isolated as previously described ${ }^{19,20}$ and analyzed to detect splicing abnormalities. Patients with type III BS and GS with homozygous or compound heterozygous mutations in CLCNKB or SLC12A3 were included in this study, whereas patients with only heterozygous mutations were excluded.

\section{Statistical analyses}

Data are expressed as mean \pm SD. All analyses were performed using standard statistical software (JMP version 10 for Windows; SAS Institute, Cary, NC). The clinical backgrounds of the patients were compared using the Mann-Whitney $U$-, Kruskal-Wallis, Steel-Dwass, Fisher's exact, Pearson's $\chi^{2}$, and Student's $t$-tests, as appropriate. A $P$ value $<0.05$ was considered statistically significant.

\section{RESULTS}

\section{Clinical and laboratory data for 163 patients with type III BS, GS, or p-BS/GS}

In total, 190 patients were referred to our hospital for mutational analysis from November 2006 to April 2014. We performed genetic tests based on the BS/GS genetic analysis algorithm proposed by Peters et al. ${ }^{21}$ and assessed the patients' clinical findings and biochemical parameters. Among these 190 patients, 21 with genetically defined type I, II, or IV BS were excluded from this study, together with 6 patients with only one mutant allele in CLCNKB or SLC12A3. Finally, 163 patients with either type III BS $(n=30)$, GS $(n=90)$ or p-BS/GS $(n=43)$ were included in this study (Figure 1). The characteristics of the three groups are summarized in Table 1. Patients with type III BS had a significantly lower mean age at diagnosis than did patients with GS or p-BS/GS $(4.2 \pm 14.0$ vs. $18.0 \pm 17.1$ vs. $36.7 \pm 16.5$ years, respectively; $P<0.001)$ (Figure $2 a)$. Thirty-eight $(88.4 \%)$ of the patients with $\mathrm{p}$-BS/GS were diagnosed during adulthood $(\geq 18$ years of age), whereas $28(93.3 \%)$ of the patients with type III BS were diagnosed during the first 3 years of life. Patients with GS had a wide range of ages at diagnosis, ranging from infancy to old age; 26 (28.9\%) were diagnosed during adulthood. These results suggest that patients clinically diagnosed with BS/GS in adulthood are more likely to have p-BS/GS or GS. However, 6

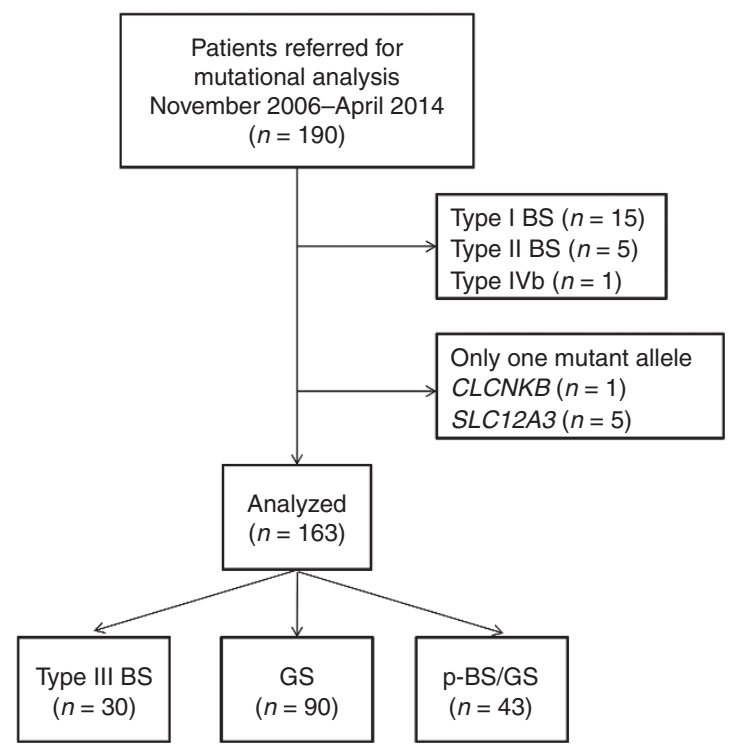

Figure 1 Flow diagram showing patient recruitment and analysis procedure. A total of 163 patients were finally enrolled in this study. BS, Bartter syndrome; GS, Gitelman syndrome. 
Table 1 Clinical and laboratory data for 163 patients with type III Bartter syndrome (BS), Gitelman syndrome (GS), or pseudo-BS/GS

\begin{tabular}{|c|c|c|c|c|}
\hline & Type III BS $(n=30)$ & $\mathrm{GS}(n=90)$ & p-BS/GS $(n=43)$ & $P$ value \\
\hline Age at diagnosis (years) & $4.2 \pm 14.0$ & $18.0 \pm 17.1$ & $36.7 \pm 16.5$ & $<0.001^{a}$ \\
\hline Male patients, $n(\%)$ & $12(40 \%)$ & $49(54.4 \%)$ & $11(25.6 \%)$ & $0.006^{\mathrm{b}}$ \\
\hline Blood $\mathrm{HCO}_{3}{ }^{-}$concentration (mEq/l) & $35.5 \pm 10.7$ & $30.8 \pm 4.30$ & $32.0 \pm 7.70$ & 0.17 \\
\hline Serum potassium concentration (mEq/l) & $2.38 \pm 0.56$ & $2.45 \pm 0.40$ & $2.42 \pm 0.41$ & 0.74 \\
\hline Serum creatinine concentration (mg/dl) & $0.49 \pm 0.38$ & $0.48 \pm 0.21$ & $1.03 \pm 0.57$ & $<0.001^{\mathrm{e}}$ \\
\hline eGFR (ml/min/1.73 m²) & $99.3 \pm 36.2$ & $116.9 \pm 25.2$ & $71.2 \pm 38.1$ & $<0.001^{f}$ \\
\hline Urinary calcium-to-creatinine ratio (mg/mg) & $0.26 \pm 0.36$ & $0.02 \pm 0.03$ & $0.18 \pm 0.40$ & $<0.001^{9}$ \\
\hline Plasma renin activity (ng/ml/h) & $100.7 \pm 118.3$ & $20.0 \pm 24.8$ & $24.8 \pm 24.6$ & $<0.001^{\mathrm{h}}$ \\
\hline Plasma aldosterone concentration (pg/ml) & $671.1 \pm 676.4$ & $238.9 \pm 256.7$ & $548.6 \pm 853.8$ & $<0.001^{i}$ \\
\hline
\end{tabular}

Values are represented as mean \pm SD unless otherwise indicated.

eGFR, estimated glomerular filtration rate;

aType III BS versus GS: $P<0.001$; type III BS versus $p-B S / G S: P<0.001$; GS versus $p-B S / G S: P<0.001$. ${ }^{b} G S$ versus $p-B S / G S: P=0.002$. Type III BS versus GS: $P<0.001$; type III $B S$ versus $p-B S / G S: P=0.004$. ${ }^{\circ}$ Type III BS versus GS: $P<0.001 ; G S$ versus $p-B S / G S: P=0.002$. ${ }^{\text {Type }}$ III BS versus $p-B S / G S: P<0.001 ; G S$ versus $p-B S / G S: P<0.001$. ${ }^{\circ}$ Type III BS versus GS: $P=0.04$; type III BS versus $\mathrm{p}-\mathrm{BS} / \mathrm{GS}: P=0.01$; GS versus $\mathrm{p}-\mathrm{BS} / \mathrm{GS}: P<0.001 .{ }^{9}$ Type III BS versus GS: $P<0.001$; GS versus $p-B S / G S: P<0.001 .{ }^{\circ}$ Type III BS versus GS: $P<0.001$; type III BS versus $p-B S / G S: P=0.001$. Type III BS versus $G S: P<0.001$.

patients with GS (6.7\%) were diagnosed at $\leq 3$ years of age, and $14(15.6 \%)$ were at $\leq 5$ years of age. Genetic testing was required to differentiate between GS and type III BS in these patients.

As expected, patients with GS had a significantly lower mean serum magnesium concentrations ( $1.58 \pm 0.35$ vs. $1.88 \pm 0.32$ vs. $1.95 \pm 0.73 \mathrm{mg} / \mathrm{dl}$, respectively; $P<0.001$ ) (Figure $2 \mathbf{b}$ ) and urinary calcium-to-creatinine ratio $(0.02 \pm 0.03$ vs. $0.26 \pm 0.36$ vs. $0.18 \pm 0.40 \mathrm{mg} / \mathrm{mg}$, respectively; $P<0.001$ ) (Figure 2c) than did patients with type III BS or p-BS/GS. Although hypomagnesemia and hypocalciuria were predominant biochemical findings in patients with GS, 5 patients with type III BS (16.7\%) and 10 with p-BS/GS (23.3\%) also showed hypomagnesemia and hypocalciuria (Supplementary Figures S1 and S2 online). However, only 41 patients (45.6\%) with GS presented with both hypomagnesemia and hypocalciuria (Supplementary Figure S3 online), and 9 showed neither hypomagnesemia nor hypocalciuria. Six patients (20.0\%) with type III BS and four (9.3\%) with $\mathrm{p}-\mathrm{BS} / \mathrm{GS}$ presented with hypercalciuria $(>0.37 \mathrm{mg} / \mathrm{mg})$. Patients with type III BS also had significantly higher blood $\mathrm{pH}$ and plasma renin activity than did those with GS or p-BS/ GS (Table 1). All three groups included some patients with a low eGFR $\left(<90 \mathrm{ml} / \mathrm{min} / 1.73 \mathrm{~m}^{2}\right)$, but the mean eGFR was significantly lower in the $\mathrm{p}$-BS/GS group than in the type III BS and GS groups $(71.2 \pm 38.1$ vs. $99.3 \pm 36.2$ vs. $116.9 \pm 25.2 \mathrm{ml} /$ $\min / 1.73 \mathrm{~m}^{2}$, respectively; $P<0.001$ ) (Figure $2 \mathrm{~d}$ ). Twelve patients with type III BS $(40.0 \%)$ presented with chronic kidney disease (stage II, $n=7$; stage III, $n=5$ ), compared with 11 patients (12.0\%) with GS (stage II, $n=9$; stage III, $n=2$ ) and 27 (62.8\%) with p-BS/GS (stage II, $n=8$; stage III, $n=13$; stage $\mathrm{IV}, n=6)$.

We next aimed to identify the clinical indices that distinguish a diagnosis of GS according to age at diagnosis. We divided all 90 patients with GS into the following three groups by age at diagnosis: childhood ( $\leq 12$ years of age), $n=55$; adolescence (13-17 years of age), $n=9$; and adulthood ( $\geq 18$ years), $n=26$. Patients with GS diagnosed during adulthood had a significantly higher urinary calcium-to-creatinine ratio $(0.03 \pm 0.03 \mathrm{mg} / \mathrm{mg})$ than did patients diagnosed during childhood or adolescence $(0.02 \pm 0.03$ vs. $0.01 \pm 0.01 \mathrm{mg} / \mathrm{mg}$, respectively; $P=0.035)$, whereas there were the no significant differences in the mean serum magnesium concentration or other parameters.

\section{Clinical and laboratory data for adult patients with type III $B S$, GS, or $p-B S / G S$}

Most patients with $\mathrm{p}-\mathrm{BS} / \mathrm{GS}$ were diagnosed during adulthood. We therefore compared adult patients with adult-diagnosed p-BS/GS and adult-diagnosed GS to adjust for the age of these two groups. The clinical features and laboratory data for these two groups are shown in Supplementary Table S1 online.

The $\mathrm{p}-\mathrm{BS} / \mathrm{GS}$ group included a significantly higher percentage of women (76.3 vs. $44.8 \%$; $P=0.008$ ), had a lower mean BMI $\left(17.9 \pm 4.1\right.$ vs. $\left.21.2 \pm 3.5 \mathrm{~kg} / \mathrm{m}^{2} ; P<0.001\right)$ (Figure $\left.3 \mathrm{a}\right)$, and had a lower mean eGFR $\left(65.1 \pm 36.2\right.$ vs. $99.8 \pm 31.2 \mathrm{ml} / \mathrm{min} / 1.73 \mathrm{~m}^{2}$; $P<0.001$ ) (Figure 3b) than the GS group. Furthermore, adult patients with $\mathrm{p}$-BS/GS had a significantly higher mean serum magnesium concentration $(1.95 \pm 0.75$ vs. $1.57 \pm 0.37 \mathrm{mg} / \mathrm{dl}$; $P=0.01$ ) (Figure $3 \mathrm{c}$ ) and urinary calcium-to-creatinine ratio $(0.07 \pm 0.08$ vs. $0.03 \pm 0.03 \mathrm{mg} / \mathrm{mg} ; P=0.008)$ (Figure $3 \mathrm{~d}$ ) than did those with GS. Although the age at diagnosis and current age were similar in both groups, the eGFR was significantly lower in patients with $\mathrm{p}$-BS/GS, suggesting the possibility of renal damage associated with hypovolemia (caused by underlying factors such as dehydration or nutritional deficiency), reflected by the higher percentage of women and lower BMI in 
a

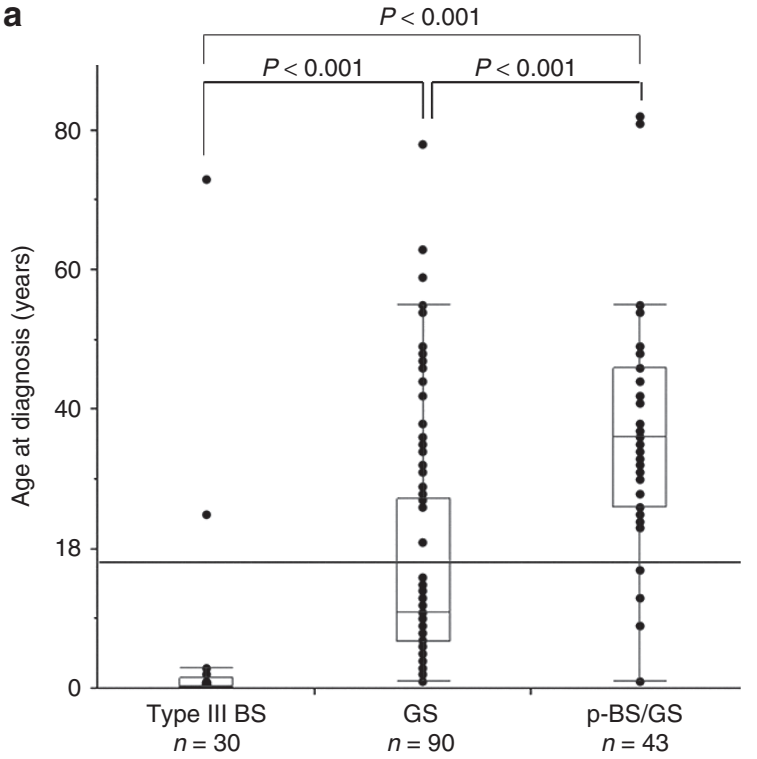

c

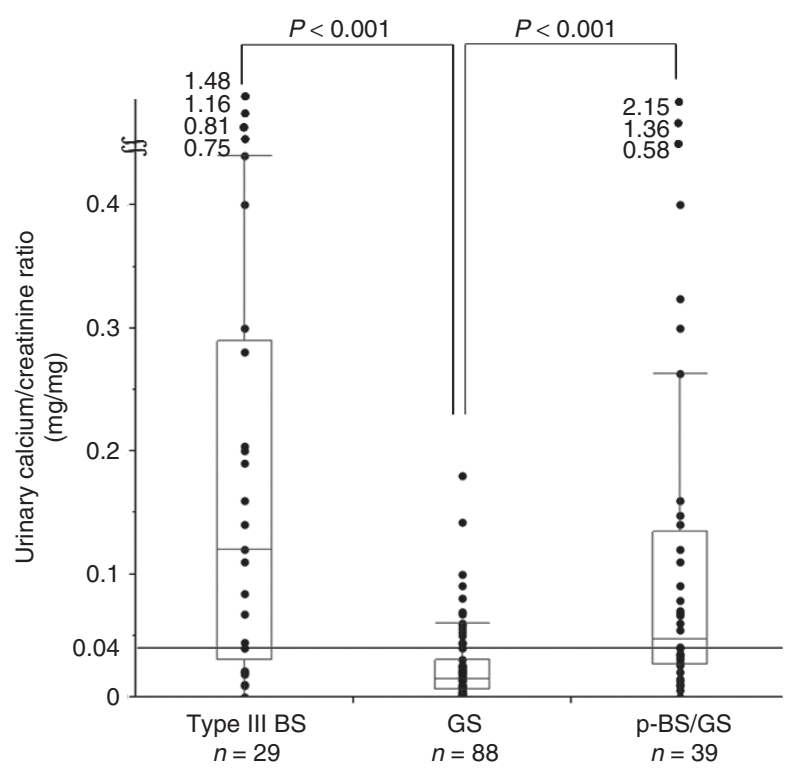

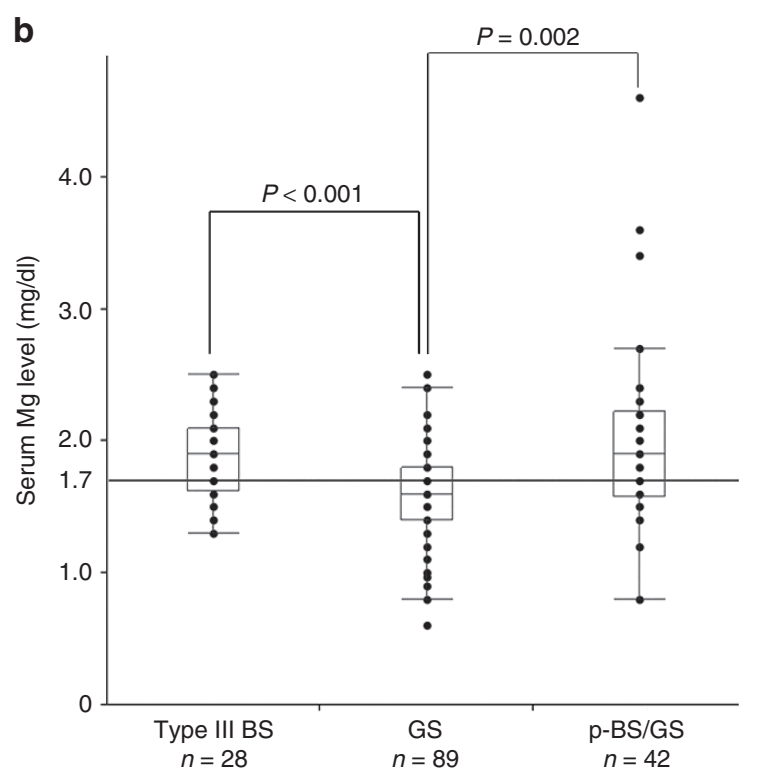

d

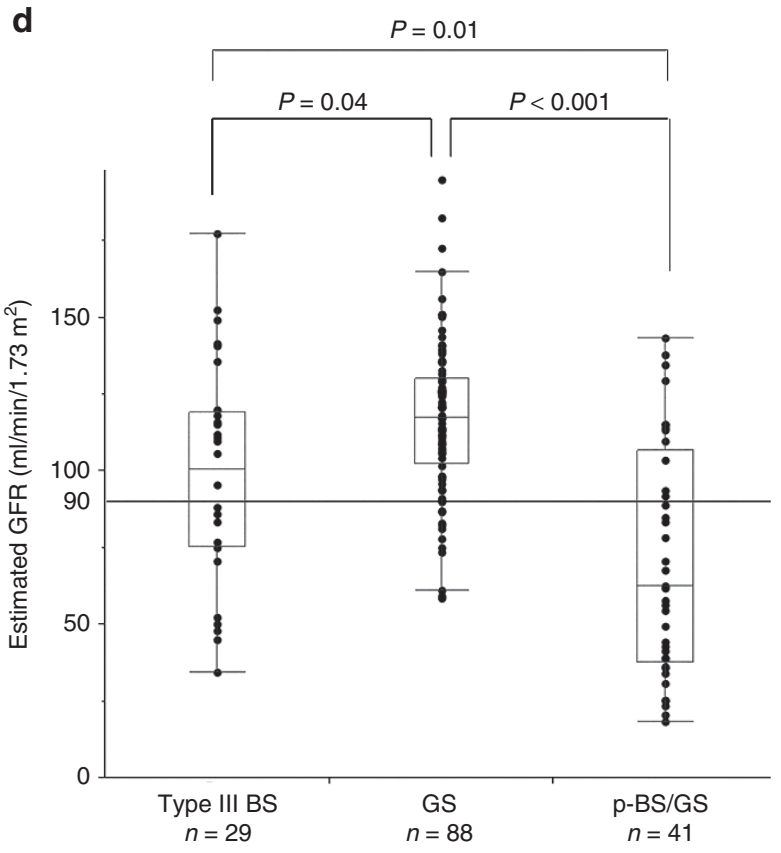

Figure 2 Selected clinical and biochemical parameters in three disorders. (a) Age at diagnosis. Patients with pseudo-Bartter syndrome/Gitelman syndrome (p-BS/GS) were significantly older at diagnosis than were patients with type III Bartter syndrome (BS) or Gitelman syndrome (GS) (36.7 \pm 16.5 vs. $4.2 \pm 14.0$ vs. $18.0 \pm 17.1$ years, respectively; $P<0.001$ ). (b) Serum magnesium concentrations. Patients with GS had a significantly lower mean serum magnesium concentration than patients with type III BS or $\mathrm{p}-\mathrm{BS} / \mathrm{GS}(1.58 \pm 0.35$ vs. $1.88 \pm 0.32$ vs. $1.95 \pm 0.73 \mathrm{mg} / \mathrm{dl}$, respectively; $P<0.001)$. Four patients lacked data. (c) Urinary calcium-to-creatinine ratio. Patients with GS had a significantly lower mean urinary calcium-to-creatinine ratio than patients with type III BS or p-BS/GS $(0.02 \pm 0.03$ vs. $0.26 \pm 0.36$ vs. $0.18 \pm 0.40 \mathrm{mg} / \mathrm{mg}$, respectively; $P<0.001)$. Six patients lacked data. (d) Estimated glomerular filtration rate (eGFR). Patients with p-BS/GS had a significantly lower mean eGFR than patients with type III BS or p-BS/GS (71.2 $\pm 38.1 \mathrm{vs} .99 .3 \pm 36.2 \mathrm{vs} .116 .9 \pm 25.2 \mathrm{ml} / \mathrm{min} / 1.73 \mathrm{~m}{ }^{2}$, respectively; $P<0.001)$. Twelve patients (40.0\%) with type III BS presented with chronic kidney disease (stage II, $n=7$; stage III, $n=5$ ), compared with 11 patients (12.0\%) with GS (stage II, $n=9$; stage III, $n=2$ ) and 27 (62.8\%) with $\mathrm{p}-\mathrm{BS} / \mathrm{GS}$ (stage II, $n=8$; stage III, $n=13$; stage IV, $n=6$ ). Six patients lacked data.

this group. Notably, 11 patients (12\%) with GS presented with a reduced eGFR $\left(<90 \mathrm{ml} / \mathrm{min} / 1.73 \mathrm{~m}^{2}\right)$ in adulthood, though the prognosis for renal function in patients with GS is usually considered benign. When we compared the adult patients with p-BS/GS with the adult patients with type III BS to adjust for age, we found no statistically significant difference in the
eGFR (Supplementary Figure S4 online), although it was significantly lower than that of adult patients with GS (Figure 3b). Excluding the influence of the aging process on the eGFR evaluation, these data show that patients with $\mathrm{p}$-BS/GS had more severe kidney dysfunction than patients with GS, but this difference was not observed in patients with type III BS. These 


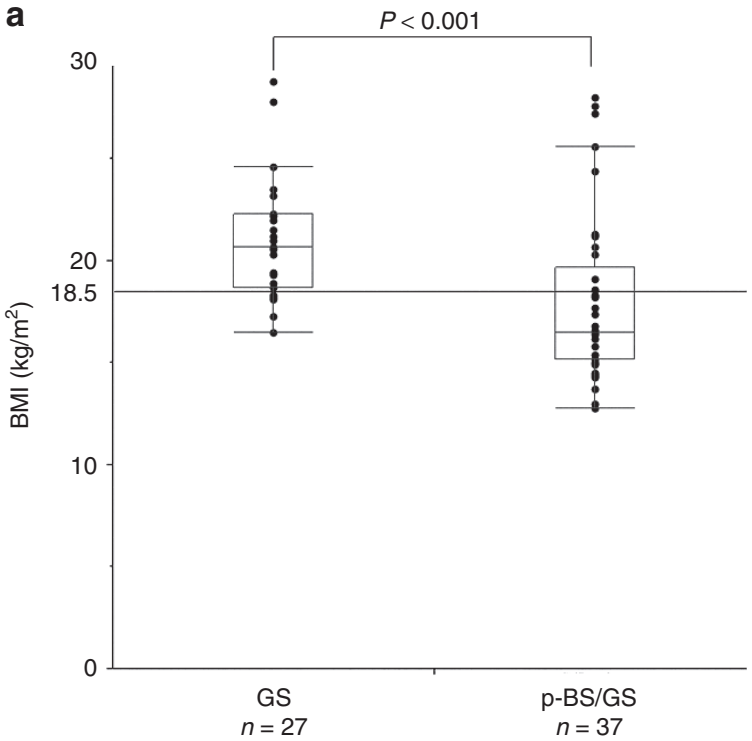

C

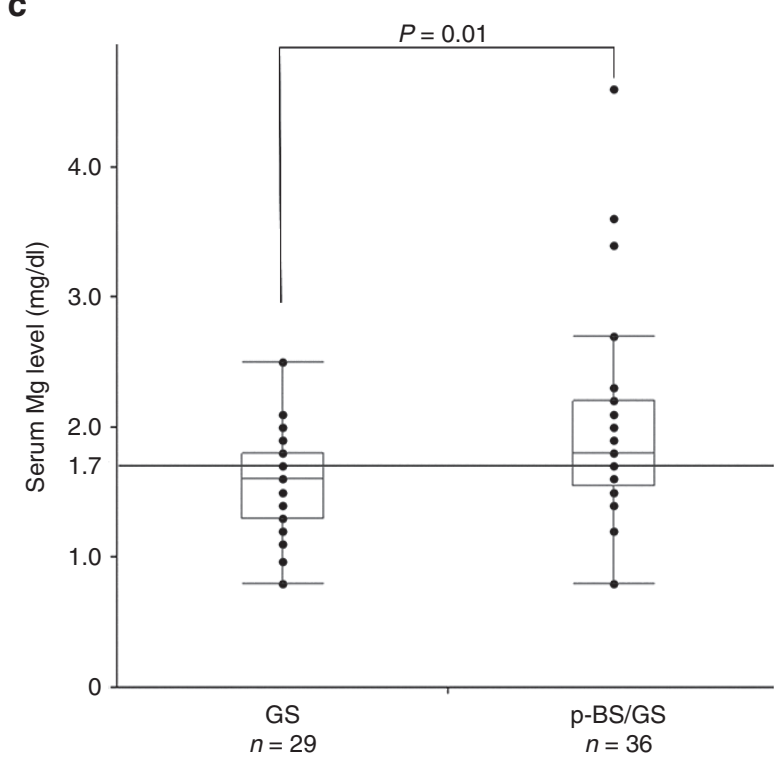

b

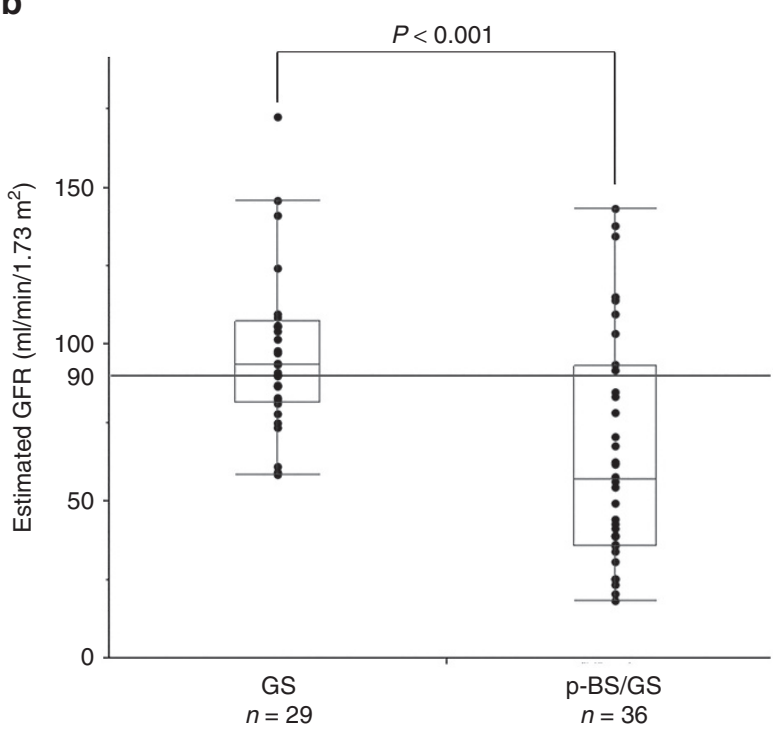

d

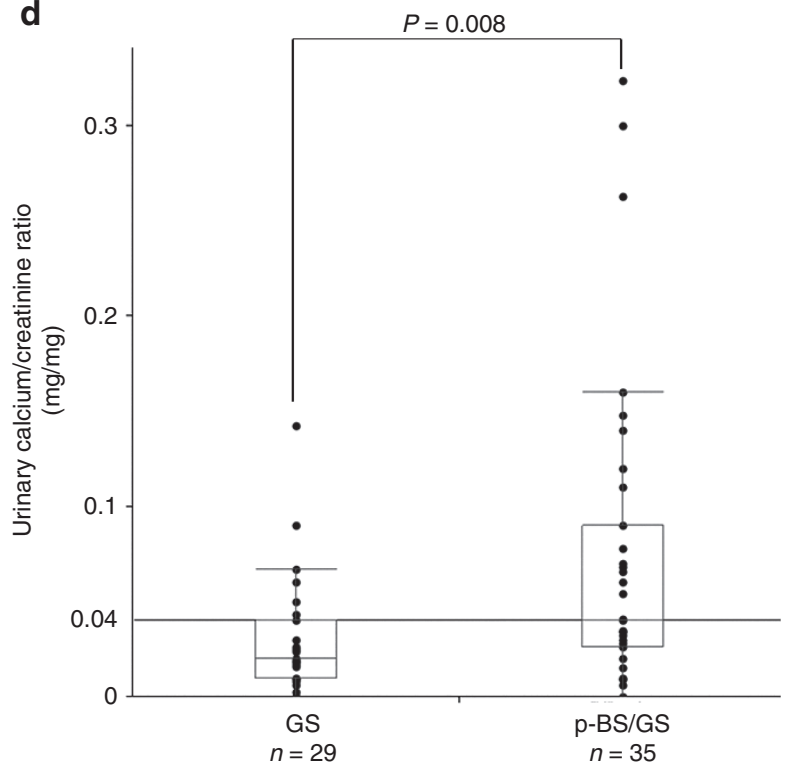

Figure 3 Selected clinical and biochemical parameters in adult Gitelman and pseudo-Bartter/Gitelman patients. (a) Body mass index (BMI) in patients aged $\geq 18$ years with Gitelman syndrome (GS) and pseudo-Bartter syndrome (BS)/Gitelman syndrome (p-BS/GS). Patients with p-BS/GS had a significantly lower mean BMI than patients with GS $\left(17.9 \pm 4.1\right.$ vs. $21.2 \pm 3.5 \mathrm{~kg} / \mathrm{m}^{2}$, respectively; $\left.P<0.001\right)$. Three patients lacked data. (b) Estimated glomerular filtration rate (eGFR) in patients with $\mathrm{GS}$ or $\mathrm{p}-\mathrm{BS} / \mathrm{GS}$ aged $\geq 18$ years. Patients with $\mathrm{p}-\mathrm{BS} / \mathrm{GS}$ had a significantly lower mean eGFR than patients with GS (65.1 $\pm 36.2 \mathrm{vs}$. $99.8 \pm 31.2 \mathrm{ml} /$ $\mathrm{min} / 1.73 \mathrm{~m}^{2}$, respectively; $P<0.001$ ). Two patients lacked data. (c) Serum magnesium concentrations in patients with $\mathrm{GS}$ or $\mathrm{p}-\mathrm{BS} / \mathrm{GS}$ aged $\geq 18$ years. Patients with $\mathrm{p}-\mathrm{BS} / \mathrm{GS}$ had a significantly higher mean serum magnesium concentration than patients with GS $(1.95 \pm 0.75 \mathrm{vs}$. $1.57 \pm 0.37 \mathrm{mg} / \mathrm{dl}$, respectively; $P=0.01)$. Two patients lacked data. (d) Urinary calcium-to-creatinine ratios in patients with GS and p-BS/GS aged $\geq 18$ years. Patients with $\mathrm{p}$-BS/GS had a significantly higher urinary calcium-to-creatinine ratio than patients with GS $(0.07 \pm 0.08 \mathrm{vs} .0 .03 \pm 0.03 \mathrm{mg} / \mathrm{mg}$, respectively; $P=0.008)$. Three patients lacked data.

findings may reflect the fact that patients with type III BS often develop kidney dysfunction. ${ }^{22}$

\section{Clinical and laboratory data for patients with p-BS/GS}

The underlying factors in all 43 patients with $\mathrm{p}$-BS/GS are shown in Supplementary Figure S5 online. Twenty-four patients (56\%) had apparent underlying causes of hypokalemia and metabolic alkalosis, including diuretic or laxative abuse $(n=14)$, severe hyperemesis gravidarum $(n=3)$, alcoholism
( $n=2)$, anorexia $(n=2)$, excessive dieting $(n=1)$, a habit of taking sweat-baths (saunas) $(n=1)$, and a habit of drinking Chinese tea, which contains diuretics $(n=1)$. Consistent with the findings of a previous study, ${ }^{5}$ most of these factors could potentially lead to hypovolemia. By contrast, no clear underlying factors were identified in the remaining 19 patients (44\%) with $\mathrm{p}-\mathrm{BS} / \mathrm{GS}$, despite detailed interviews. The 38 patients with $\mathrm{p}$-BS/GS who were aged $\geq 18$ years were divided into two groups according to the presence or absence of underlying 
factors. The clinical differences between these groups are shown in Supplementary Table S2 online. The only significant difference was in height, which was significantly shorter in the group without underlying factors. This suggests that $\mathrm{p}-\mathrm{BS} / \mathrm{GS}$ in patients without obvious underlying factors might be partially caused by genetic factors different from those responsible for BS or GS because a short stature reflects underlying factors that existed during the growth phase.

We focused on the patients with p-BS/GS caused by laxative abuse because laxative abuse is the most common cause of p-BS/ GS (Supplementary Table S3 online, Supplementary Figure S6 online). Eleven patients had p-BS/GS caused by laxative abuse, including seven who used magnesium oxide laxatives and four who used laxatives that did not contain magnesium (sennoside, $n=3$; Japanese traditional medicine, $n=1$ ). Four of the five patients with $\mathrm{p}-\mathrm{BS} / \mathrm{GS}$ with markedly high serum magnesium concentrations $(>2.5 \mathrm{mg} / \mathrm{dl})$ had a history of chronic use of a magnesium oxide laxative preparation. Unexpectedly, none of the patients who used laxatives that did not contain magnesium had hypomagnesemia (Supplementary Figure S6 online). On the basis of these results we can say that patients with pseudo-BS/GS caused by laxatives tend to have normal or high serum magnesium concentrations.

We also compared the 11 patients with p-BS/GS caused by laxative abuse with the 29 adult patients with GS (Supplementary Table S3 online). As expected, the patients with p-BS/GS caused by laxative abuse had a lower mean BMI $(18.7 \pm 4.8$ vs. $21.2 \pm 3.5 \mathrm{~kg} / \mathrm{m}^{2}$, respectively; $\left.P=0.02\right)$ and a lower mean eGFR $\left(64.8 \pm 25.8\right.$ vs. $99.8 \pm 31.2 \mathrm{ml} / \mathrm{min} / 1.73 \mathrm{~m}^{2}$, respectively; $P=0.005)$ than the adult patients with GS. Notably, the patients with $\mathrm{p}-\mathrm{BS} / \mathrm{GS}$ caused by laxative abuse had significantly higher plasma renin activity $(26.2 \pm 17.9$ vs. $15.9 \pm 9.9 \mathrm{ng} / \mathrm{ml} / \mathrm{h}$, respectively; $P=0.03$ ) and plasma aldosterone concentrations $(617.4 \pm 655.8$ vs. $273.4 \pm 333.8 \mathrm{pg} / \mathrm{ml}$, respectively; $P=0.02)$ than adult patients with GS, reflecting the marked activation of the renin-aldosterone system secondary to chronic diarrhea. Furthermore, patients with p-BS/GS caused by laxative abuse had a significantly higher mean urinary calcium-to-creatinine ratio than patients with GS $(0.09 \pm 0.06$ vs. $0.03 \pm 0.03 \mathrm{mg} / \mathrm{mg}$, respectively; $P=0.04$ ). These results may clarify the characteristics of $\mathrm{p}-\mathrm{BS} / \mathrm{GS}$ caused by laxative abuse.

Four patients with p-BS/GS had hypercalciuria. Among them, one (a 1-year-old girl) was being given a Chinese tea containing a diuretic, possibly a loop diuretic, by her parents. The other three patients were relatively young (two were 9 years old and one was 13 years old), which might suggest that they had a hereditary disorder other than BS/GS. None of the patients in our cohort were taking loop diuretics for medical purposes.

\section{FENa and FECl}

Impaired tubular reabsorption of sodium chloride, resulting in urinary loss, is a common pathophysiological mechanism of BS/ GS. We therefore compared FENa and $\mathrm{FECl}$ among the patients with BS, GS, and p-BS/GS with available urine sodium and/ or chloride concentration data. Patients with type III BS had a significantly higher mean FENa (1.62 $\pm 0.79 \%$ vs. $0.69 \pm 0.44 \%$ vs. $0.32 \pm 0.28 \%$, respectively; $P<0.001$ ) (Figure $4 a$ ) and $\mathrm{FECl}$ $(2.80 \pm 1.44 \%$ vs. $1.10 \pm 0.68 \%$ vs. $0.44 \pm 0.45 \%$, respectively; $P<0.001$ ) (Figure 4b) than patients with GS and p-BS/GS. These results suggest that patients with genetically defined BS/ GS show more severe defects in the tubular reabsorption of sodium chloride than patients with $\mathrm{p}-\mathrm{BS} / \mathrm{GS}$.
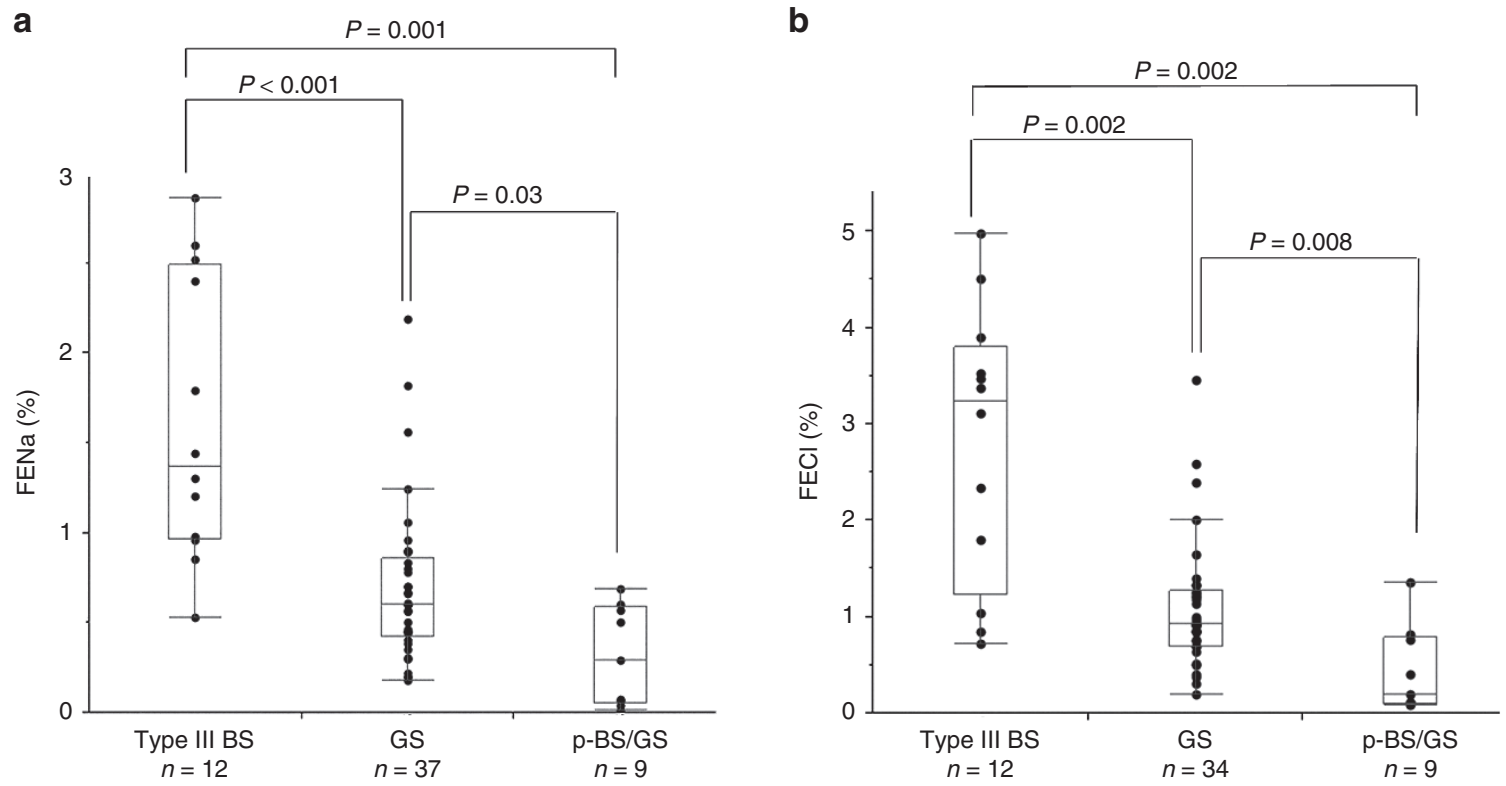

Figure 4 Fractional excretion of sodium and chloride in three disorders. (a) Fractional excretion of sodium (FENa). Patients with type III Bartter syndrome (BS) had a significantly higher FENa than patients with Gitelman syndrome (GS) or pseudo-BS/GS (p-BS/GS) $(1.62 \pm 0.79 \%$ vs. $0.69 \pm 0.44 \%$ vs. $0.32 \pm 0.28 \%$, respectively; $P<0.001)$. (b) Fractional excretion of chloride (FECI). Patients with type III BS had a significantly higher FECI than patients with $\mathrm{GS}$ or $\mathrm{p}$-BS/GS $(2.80 \pm 1.44 \%$ vs. $1.10 \pm 0.68 \%$ vs. $0.44 \pm 0.45 \%$, respectively; $P<0.001)$. 


\section{DISCUSSION}

This study demonstrates for the first time the clinical distinctions among patients with genetically defined type III BS, GS, and p-BS/GS. The results provide useful and novel clinical information about these three diseases, which are usually difficult to differentiate and are frequently confused with one another in the clinical setting.

BS and GS have been divided into three main categories according to age at onset: antenatal BS (types I, II, IV, and IVb), classic BS (type III BS), and GS. Antenatal BS is usually easy to diagnose because patients present with typical symptoms, such as polyhydramnios, low birth weight, or failure to thrive, during the antenatal or neonatal period. ${ }^{5,21}$ By contrast, the clinical manifestations of type III BS and GS may vary and resemble each other. ${ }^{711-13}$ Furthermore, $\mathrm{p}$-BS/GS is difficult to distinguish from type III BS or GS because its clinical features match those of the latter two diseases. Correctly distinguishing these three diseases based on their clinical characteristics is thus important. We therefore analyzed and compared the clinical data for 163 Japanese patients with genetically defined type III BS, GS, and $\mathrm{p}-\mathrm{BS} / \mathrm{GS}$.

Age at diagnosis differed significantly among patients with the three diseases. A previous report suggested that patients with type III BS often presented with the disease during childhood, whereas patients with GS were usually diagnosed during late childhood or adulthood. ${ }^{23}$ In this study most patients with type III BS (93\%) were diagnosed before the age of 3 years, whereas most patients with p-BS/GS (88\%) were diagnosed during adulthood. About one-third (29\%) of patients with GS were diagnosed during adulthood. These findings suggest that age at diagnosis may be useful for differentiating among these three diseases. Distinguishing between infantile-onset GS and type III BS remains problematic, however, and genetic testing is required to determine the nature of the disease in these patients.

We also found that patients in all three groups demonstrated varying degrees of progressive renal impairment, as previously reported. ${ }^{22,24-26}$ Tseng et al..$^{24}$ reported that 7 of 117 patients (6\%) with genetically diagnosed GS developed chronic kidney disease (stage III or IV). In this study 12 of 30 patients (40.0\%) with type III BS, 11 of 90 (12.0\%) with GS, and 27 of 43 (62.8\%) with $\mathrm{p}$-BS/GS presented with stage II-IV chronic kidney disease. Thus caution is necessary when establishing a list of differential diagnoses because both hypokalemic metabolic alkalosis and renal impairment are common to all three diseases.

We further clarified the differences between adult patients with p-BS/GS and adult patients with GS to adjust for the age of these two groups and found that $\mathrm{p}-\mathrm{BS} / \mathrm{GS}$ was more common among adult women with a lower BMI and eGFR. Previous reports demonstrated that dehydration caused by anorexia and diuretic or laxative abuse causes renal hypoperfusion, resulting in a reduced glomerular filtration rate. ${ }^{27,28}$ These reports support our current data, given that anorexia and diuretic and laxative abuse are generally recognized to be more common among women.
The results of this study also confirmed the significant phenotypic overlap in serum magnesium concentrations and urinary calcium-to-creatinine ratios among the three diseases. Some cases of type III BS were previously reported to show features similar to those of GS, including hypomagnesemia and hypocalciuria. ${ }^{711-13}$ In addition, one report published in 2003 showed that, in a large consanguineous family, both the BS and GS phenotypes were present with the same CLCNKB mutation. ${ }^{13}$ In the same year a review article pointed out the phenotypic overlaps between type III BS and GS. ${ }^{29}$ In this study $16.7 \%$ of patients with type III BS and $23.3 \%$ with p-BS/GS had hypomagnesemia and hypocalciuria, as in GS. Interestingly, only $47 \%$ of patients with GS typically presented with hypomagnesemia and hypocalciuria. These data demonstrate the difficulty of distinguishing among these disorders on the basis of the absence or presence of hypomagnesemia and hypocalciuria.

We also attempted to identify the underlying factors contributing to the development of p-BS/GS. Among the 43 patients with p-BS/GS, more than half (56\%) had contributing factors, including diuretic or laxative abuse in 33\%. The remainder (44\%), however, had no clear contributing factors. Although the acquisition of a detailed history has been considered to allow for discernment between GS and p-BS/GS, the limited value of this approach was demonstrated by the low detection rate of underlying factors in this study. Veldhuis et al. ${ }^{30}$ reported that measurement of the urinary chloride concentration is useful for differential diagnosis based on urinary or fecal chloride loss. We therefore evaluated urinary electrolyte excretion (FENa and $\mathrm{FECl}$ ). In this study patients with type III BS and GS had significantly higher mean FENa and FECl values than patients with p-BS/GS. These findings suggest that FENa and FECl may be useful parameters for differentiating among these three diseases.

There are several possible reasons for the failure to identify underlying factors. We may have failed to detect mutations in the CLCNKB or SLC12A3 gene; however, that we would have missed both mutations on the paternal and maternal alleles is unlikely. We failed to detect two mutations in only six patients, and if our strategy was inadequate, we expect this number would have been much larger. Some patients with p-BS/GS also may have presented with inherited disorders other than BS/ GS. Choi et al. ${ }^{31}$ recently reported that SLC26A3 gene mutations were responsible for congenital chloride diarrhea in 5 of 39 patients with clinically diagnosed BS. As reported previously, however, we failed to detect any SLC26A3 gene mutations in patients with p-BS/GS with no detectable underlying factors. ${ }^{32}$ Our findings suggest that SLC26A3 mutations may have been quite rare in our cohort. We also demonstrated that adult patients with $\mathrm{p}-\mathrm{BS} / \mathrm{GS}$ with no underlying factors had a significantly shorter stature than those with underlying factors. This suggests the possible existence of unidentified inherited disorders in patients with $\mathrm{p}-\mathrm{BS} / \mathrm{GS}$. In fact, some researchers doubt the existence of new loci other than those on genes already identified for these phenotypes. ${ }^{29,31}$ Identification of defects in other transporter genes may significantly improve 
our understanding of the underlying mechanisms of renal salt homeostasis. Next-generation sequencing, in addition to functional studies and in vivo analysis using transgenic animals, is needed to characterize these undiagnosed cases. This new technology will also reduce the burden of genetic analysis and allow for the detection of unidentified mutations.

In this study five patients were heterozygous carriers of NCCT mutations. We could not detect the other mutation despite performing several types of genetic analyses. For accurate phenotyping of GS, we excluded these patients from this study. However, some previous reports have indicated the possibility that a single loss-of-function mutation of the NCCT gene can result in a phenotype similar to that of patients treated by low-dose thiazide diuretics. ${ }^{33}$ Recent large cohort studies also showed that about $8 \%$ of patients with GS have only heterozygous mutations in the NCCT gene; that GS occurs by heterozygous mutations in NCCT-gene haploinsufficiency or dominant-negative effects by hypomorphic alleles remains possible. ${ }^{12,24}$ Further study is needed to answer this long-standing question.

This study had several limitations. First, we diagnosed p-BS/GS based on negative genetic results for CLCNKB, SLC12A3, and SLC26A3 mutations, and we may have failed to detect mutations in these genes. However, it is very unlikely that we missed mutations in both alleles, as required for autosomal recessive inheritance, as discussed above. The second limitation is that we observed the patients' clinical courses for only a relatively short period of time. Long-term follow-up is necessary to clarify their prognoses, including their renal prognoses.

In conclusion, we provided herein the first criteria for establishing clinical distinctions among genetically diagnosed type III BS, GS, and p-BS/GS. Our results indicate that p-BS/GS is particularly common among adult women with lower BMIs and eGFRs. These results suggest that age at diagnosis, sex, $\mathrm{BMI}$, and renal function, in addition to the serum magnesium concentration and urinary calcium-to-creatinine ratio, should be taken into consideration for the differential diagnosis of type III BS, GS, and p-BS/GS.

\section{SUPPLEMENTARY MATERIAL}

Supplementary material is linked to the online version of the paper at http://www.nature.com/gim

\section{ACKNOWLEDGMENTS}

This study was supported by a grant from the Ministry of Health, Labour and Welfare, Japan, for Research on Rare Intractable Diseases in Kidney and Urinary Tract (H24-nanchitou (nan)-ippan-041 to Kazumoto lijima) in the "Research on Measures for Intractable Diseases" Project; a Grant-in-Aid for Scientific Research (KAKENHI) from the Ministry of Education, Culture, Sports, Science and Technology of Japan (subject ID: 25893131 to K.N.); and a grant from the Mother and Child Health Foundation (subject ID: 25-7 to K.N.).

\section{DISCLOSURE}

The authors declare no conflict of interest.

\section{REFERENCES}

1. Simon DB, Bindra RS, Mansfield TA, et al. Mutations in the chloride channel gene, CLCNKB, cause Bartter's syndrome type III. Nat Genet 1997;17:171-178.

2. Simon DB, Karet FE, Hamdan JM, DiPietro A, Sanjad SA, Lifton RP. Bartter's syndrome, hypokalaemic alkalosis with hypercalciuria, is caused by mutations in the Na-K-2Cl cotransporter NKCC2. Nat Genet 1996;13:183-188.

3. Simon DB, Karet FE, Rodriguez-Soriano J, et al. Genetic heterogeneity of Bartter's syndrome revealed by mutations in the K+ channel, ROMK. Nat Genet 1996;14:152-156.

4. Simon DB, Nelson-Williams C, Bia MJ, et al. Gitelman's variant of Bartter's syndrome, inherited hypokalaemic alkalosis, is caused by mutations in the thiazide-sensitive Na-Cl cotransporter. Nat Genet 1996;12:24-30.

5. Seyberth HW, Schlingmann KP. Bartter- and Gitelman-like syndromes: saltlosing tubulopathies with loop or DCT defects. Pediatr Nephro/ 2011;26:17891802.

6. Birkenhäger R, Otto $E$, Schürmann MJ, et al. Mutation of BSND causes Bartter syndrome with sensorineural deafness and kidney failure. Nat Genet 2001;29:310-314.

7. Nozu K, lijima K, Kanda K, et al. The pharmacological characteristics of molecular-based inherited salt-losing tubulopathies. J Clin Endocrinol Metab 2010;95:E511-E518.

8. Estévez R, Boettger T, Stein V, et al. Barttin is a Cl- channel beta-subunit crucial for renal Cl- reabsorption and inner ear K+ secretion. Nature 2001;414:558561.

9. Schlingmann KP, Konrad M, Jeck $N$, et al. Salt wasting and deafness resulting from mutations in two chloride channels. N Engl J Med 2004;350:1314-1319.

10. Nozu K, Inagaki T, Fu XJ, et al. Molecular analysis of digenic inheritance in Bartter syndrome with sensorineural deafness. J Med Genet 2008;45:182-186.

11. Lee $\mathrm{BH}, \mathrm{Cho} \mathrm{HY}$, Lee $\mathrm{H}$, et al. Genetic basis of Bartter syndrome in Korea. Nephrol Dial Transplant 2012;27:1516-1521.

12. Vargas-Poussou R, Dahan K, Kahila D, et al. Spectrum of mutations in Gitelman syndrome. J Am Soc Nephrol 2011;22:693-703.

13. Zelikovic I, Szargel R, Hawash A, et al. A novel mutation in the chloride channel gene, CLCNKB, as a cause of Gitelman and Bartter syndromes. Kidney Int 2003:63:24-32.

14. Schwartz GJ, Haycock GB, Edelmann CM Jr, Spitzer A. A simple estimate of glomerular filtration rate in children derived from body length and plasma creatinine. Pediatrics 1976;58:259-263.

15. Schwartz GJ, Gauthier B. A simple estimate of glomerular filtration rate in adolescent boys. J Pediatr 1985;106:522-526.

16. Matsuo S, Imai E, Horio M, et al.; Collaborators developing the Japanese equation for estimated GFR. Revised equations for estimated GFR from serum creatinine in Japan. Am J Kidney Dis 2009;53:982-992.

17. Fukuyama S, Okudaira S, Yamazato S, Yamazato M, Ohta T. Analysis of renal tubular electrolyte transporter genes in seven patients with hypokalemic metabolic alkalosis. Kidney Int 2003;64:808-816.

18. Nozu K, Fu XJ, Nakanishi K, et al. Molecular analysis of patients with type III Bartter syndrome: picking up large heterozygous deletions with semiquantitative PCR. Pediatr Res 2007;62:364-369.

19. Kaito H, Nozu K, Fu XJ, et al. Detection of a transcript abnormality in mRNA of the SLC12A3 gene extracted from urinary sediment cells of a patient with Gitelman's syndrome. Pediatr Res 2007;61:502-505

20. Nozu K, lijima K, Kawai K, et al. In vivo and in vitro splicing assay of SLC12A1 in an antenatal salt-losing tubulopathy patient with an intronic mutation. Hum Genet 2009;126:533-538.

21. Peters $M$, Jeck N, Reinalter $S$, et al. Clinical presentation of genetically defined patients with hypokalemic salt-losing tubulopathies. Am J Med 2002;112:183190.

22. Bettinelli A, Borsa N, Bellantuono R, et al. Patients with biallelic mutations in the chloride channel gene CLCNKB: long-term management and outcome. Am J Kidney Dis 2007:49:91-98.

23. Shaer AJ. Inherited primary renal tubular hypokalemic alkalosis: a review of Gitelman and Bartter syndromes. Am J Med Sci 2001;322:316-332.

24. Tseng MH, Yang SS, Hsu YJ, et al. Genotype, phenotype, and follow-up in Taiwanese patients with salt-losing tubulopathy associated with SLC12A3 mutation. J Clin Endocrino/ Metab 2012:97:E1478-E1482. 


\section{ORIGINAL RESEARCH ARTICLE}

25. Knoers NV, Levtchenko EN. Gitelman syndrome. Orphanet J Rare Dis 2008;3:22.

26. Calò LA, Marchini F, Davis PA, Rigotti P, Pagnin E, Semplicini A. Kidney transplant in Gitelman's syndrome. Report of the first case. J Nephrol 2003;16: 144-147.

27. Boag F, Weerakoon J, Ginsburg J, Havard CW, Dandona P. Diminished creatinine clearance in anorexia nervosa: reversal with weight gain. J Clin Pathol 1985;38:60-63.

28. Lowinger K, Griffiths RA, Beumont PJ, Scicluna H, Touyz SW. Fluid restriction in anorexia nervosa: a neglected symptom or new phenomenon? Int J Eat Disord 1999;26:392-396.

29. Zelikovic I. Hypokalaemic salt-losing tubulopathies: an evolving story. Nephrol Dial Transplant 2003;18:1696-1700.
30. Veldhuis JD, Bardin CW, Demers LM. Metabolic mimicry of Bartter's syndrome by covert vomiting: utility of urinary chloride determinations. Am J Med 1979;66:361-363.

31. Choi M, Scholl Ul, Ji W, et al. Genetic diagnosis by whole exome capture and massively parallel DNA sequencing. Proc Natl Acad Sci USA 2009;106:1909619101.

32. Ishimori S, Kaito H, Matsunoshita N, et al. SLC26A3 gene analysis in patients with Bartter and Gitelman syndromes and the clinical characteristics of patients with unidentified mutations. Kobe J Med Sci 2013;59:E36-E43.

33. Fava C, Montagnana M, Rosberg $L$, et al. Subjects heterozygous for genetic loss of function of the thiazide-sensitive cotransporter have reduced blood pressure. Hum Mol Genet 2008;17:413-418. 